\title{
Toward the holistic, reference, and extendable atlas of the human brain, head, and neck
}

\author{
Wieslaw L. Nowinski
}

Received: 25 January 2015/ Accepted: 29 January 2015/Published online: 27 February 2015

(c) The Author(s) 2015. This article is published with open access at Springerlink.com

\begin{abstract}
Despite numerous efforts, a fairly complete (holistic) anatomical model of the whole, normal, adult human brain, which is required as the reference in brain studies and clinical applications, has not yet been constructed. Our ultimate objective is to build this kind of atlas from advanced in vivo imaging. This work presents the taxonomy of our currently developed brain atlases and addresses the design, content, functionality, and current results in the holistic atlas development as well as atlas usefulness and future directions. We have developed to date 35 commercial brain atlases (along with numerous research prototypes), licensed to 63 companies and institutions, and made available to medical societies, organizations, medical schools, and individuals. These atlases have been applied in education, research, and clinical applications. Hundreds of thousands of patients have been treated by using our atlases. Based on this experience, the first version of the holistic and reference atlas of the brain, head, and neck has been developed and made available. The atlas has been created from multispectral 3 and 7 Tesla and high-resolution $\mathrm{CT}$ in vivo scans. It is fully $3 \mathrm{D}$, scalable, interactive, and highly detailed with about 3,000 labeled components. This atlas forms a foundation for the
\end{abstract}

Electronic supplementary material The online version of this article (doi:10.1007/s40708-015-0012-4) contains supplementary material, which is available to authorized users.

W. L. Nowinski $(\bowtie)$

Department of Radiology, University of Washington, Seattle,

WA, USA

e-mail: nowinski@uw.edu

URL: http://www.wieslawnowinski.com development of a multi-level molecular, cellular, anatomical, physiological, and behavioral brain atlas platform.

Keywords Brain atlas $\cdot$ Head and neck atlas · Taxonomy

\section{Introduction}

After The Decade of the Brain in the 1990s, the twenty first century is considered the century of the brain. Numerous efforts tackle modeling, mapping, and atlasing of the human brain. We recently witness unprecedented big (in terms of scale and funding) and highly publicized brain projects, such as The BRAIN Initiative (Brain Research through Advancing Innovate Neurotechnologies) [1], The Human Brain Project to build a computer model of the human brain [1,2], The Connectome Project to map structural connections [1], The Big Brain providing very high-resolution images [3], and The Allen Brain Atlas to map gene expression integrated with the underlying neuroanatomy $[4,5]$.

Despite their novelty, complexity, and brain knowledge expansion, none of these challenging initiatives addresses the basic issue that a fairly complete neuroanatomical model of the whole human brain, which is required as the reference in brain studies and clinical applications, does not exist.

The existing (commercially available) brain atlases, such as ADAM [6], Cerefy [7], Digital Anatomist [8], and Voxel-man [9], are anatomically incomplete; moreover, besides our Cerefy atlases [10], none of these atlases has been used in clinical applications.

In order to capture structural, functional, and molecular variations, numerous endeavors aim at the creation of population-based and spatiotemporal atlases [11-13], including our efforts on the probabilistic functional atlases $[10,14]$, the population-based stroke atlas [15], and the 
atlas of cerebrovascular variants [16]. However, these atlases have some limitations [17], such as fuzziness due to averaging, low parcellation, limited anatomical content, and they are often targeted at specific disease types.

Our contribution to these global, brain-related efforts is to create adult human brain atlases and develop atlas-based applications. For over two decades, we have developed 35 brain atlases, licensed to 63 companies and institutions, and made available to medical societies, organizations, medical schools, and individuals. In contrast to the ongoing initiatives, our approach is (1) guided by research, clinical, and market perspectives; (2) top-down; and (3) holistic.

The atlases developed so far by us can be grouped in three categories, namely, derived from print materials, in vivo imaging, and population (including patient-specific) data.

Our ultimate goal is to create a holistic atlas of the whole adult human brain along with the head and neck. The atlas is three-dimensional (3D), advanced, detailed, interactive, accurate, reference, realistic, high resolution, fully parcellated, completely labeled, spatially consistent, stereotactic, user friendly, extendable (scalable), composable, dissectible, explorable, and modular.

This work aims to present the taxonomy of the currently developed atlases and to address the design, content, functionality, and current results in the holistic atlas development as well as atlas usefulness and future directions.

\section{Materials and methods}

\subsection{Materials}

A normal, adult, male specimen (W.L.N.) has been scanned over many years on various scanners and with variable pulse sequences. A single specimen was employed to ensure spatial consistency and extendibility of the atlas. In order to build the brain model (along with the skin, glands and head muscles), multispectral magnetic resonance (MR) data were acquired on 1.5 Tesla (T), $3 \mathrm{~T} / 8$ channels, $3 \mathrm{~T} / 32$ channels, and $7 \mathrm{~T}$ instruments, and with T1-weighted (T1W), T2-weighted (T2W), magnetization prepared rapid gradient echo (MPRAGE), 2D time-of-flight (TOF), 3D TOF, susceptibilityweighted imaging (SWI), spoiled gradient-recalled (SPGR), and diffusion tensor imaging (DTI) pulse sequences. The skull model was created from a high-resolution computed tomography $(\mathrm{CT})$ scan. To create the neck vessels, MR data were acquired on a $3 \mathrm{~T}$ scanner with phase contrast angiography (PCA) and M2D (Philips term) inflow pulse sequences.

\subsection{Atlas taxonomy}

Several taxonomies of brain atlases have been proposed, and we also have introduced some earlier $[14,18]$. Here, we present and discuss the taxonomy of all the brain atlases created by us. This taxonomy is diagrammed in Fig. 1.

We have developed three major families of atlases derived from print materials, in vivo imaging, and population (including patient-specific) data. The print material-based family has been successful, several atlases have been developed [7, 19-22], and brain atlas libraries incorporated into surgical workstations [10]. However, despite integration of multiple atlases with complementary contents (gross anatomy [23], deep structures [24], brain connections [25], and sulcal patterns [26]) within a single system with their enhanced electronic versions [27] as well as extending these atlases to 3D [27, 28], an accurate match of the component atlases and content extension were not feasible. This was the main reason to start constructing a new generation of brain atlases, based on imaging of a single specimen. Another reason is that none of the recent big brain projects addresses a construction of a complete neuroanatomical model. Such a reference model is required in brain studies and clinical applications as well as in big projects, such as BRAIN Initiative and The Human Brain Project (see the Sect. 4).

Our endeavor shifts the brain atlas development paradigm FROM fragmented studies and mosaic content, "copy and paste" neuroanatomy, static presentation, and tedious neurological description TO holistic representation, truly 3D structural, vascular, connectional and functional neuroanatomy, dynamic brain de/composition (from blocks to brain), dysfunction brain atlases, and brain at work. Several atlases have been created within this family (an atlas of cerebral vasculature [29] as well as the human brain in 1492 [30, 31] (two editions), 1969 [32, 33] (2 editions) along with mobile versions on iPad [34] and Android-based [35], and 2953 [36] pieces along with the 3D atlas of neurologic disorder (on desktop [37] and iPad [38])), and they excellently illustrate our strategy and paradigm shift.

Several atlases have been created for neurologic disorders [39-41]. They provide disorder-localization relationships and tools for exploration as well as correlate cerebral pathology with the underlying neuroanatomy and the resulting neurological deficits. These atlases present various simulated cerebral pathologic situations (lesions) in 3D that are labeled with the resulting disorders along with the surrounding highly parcellated neuroanatomy. Disorders are linked to textbook materials and described in terms of the resulting signs, symptoms and/or syndromes (for instance in the Stroke Atlas [41], the disorders are compiled for ischemic stroke, hemorrhagic stroke, and cerebral aneurysms).

The current reference atlas is by definition deterministic, where the brain can be easily composed and decomposed, and each cerebral component is uniquely labeled, freely 


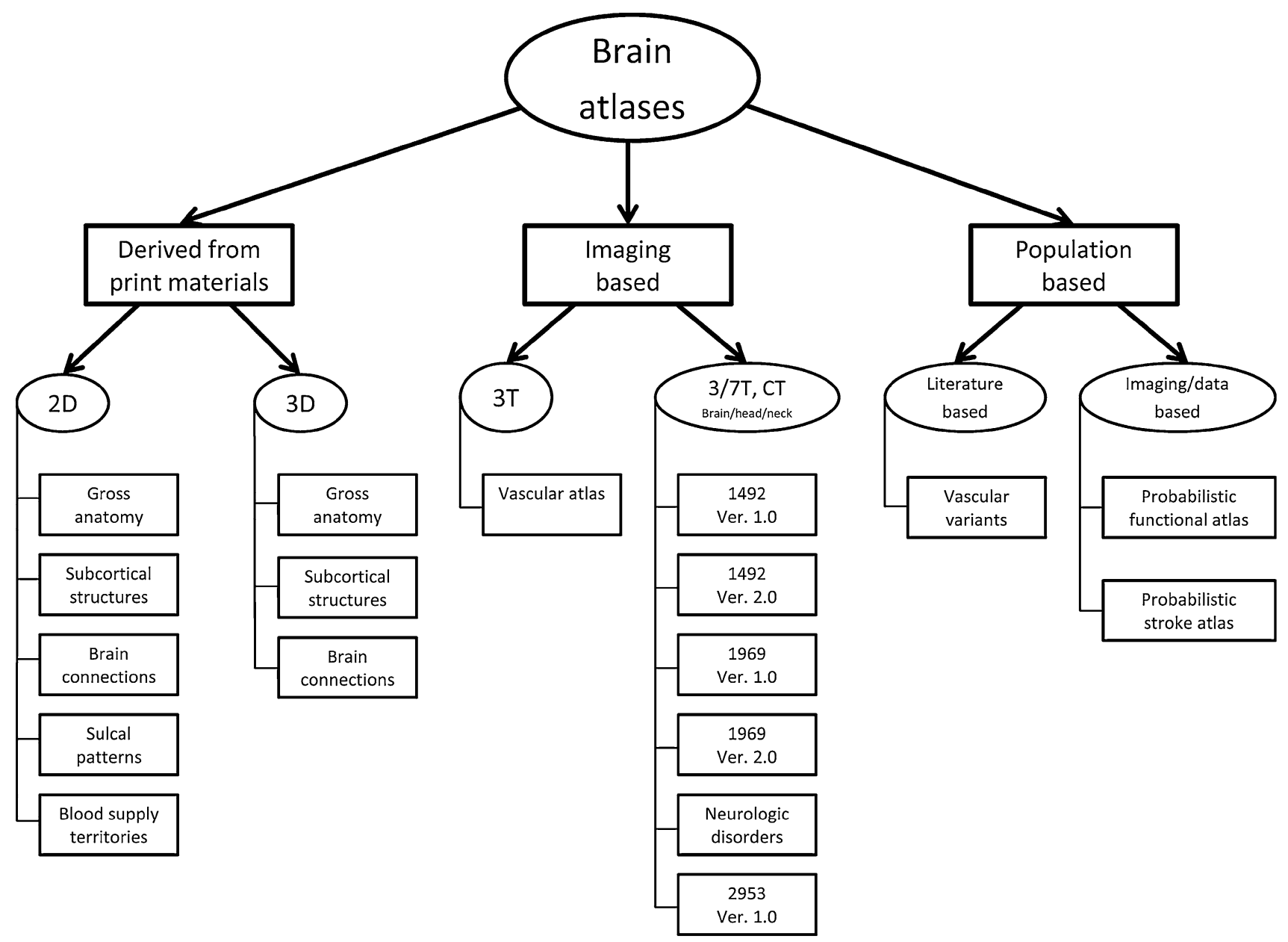

Fig. 1 Taxonomy of the currently developed brain atlases

manipulated, and presented within its user-selected surrounding context. These operations are not feasible in existing population-based atlases, which are typically mosaic type.

As a single-specimen atlas does not contain variability, the third family of atlases has been created to deal with this problem. Our current efforts to create the holistic, deterministic (a single specimen), and reference atlas have taken more than a decade and this is still work in progress. In our opinion, the development of the holistic, probabilistic, and reference atlas is an enormous and expensive task. Therefore, our concept of handling variability is to integrate it around the reference deterministic atlas. A good illustration of this approach is our atlas of arterial cerebral variants (see the Sect. 4). The third family of atlases is based on imaging and non-imaging population data. We have used patientspecific data to develop atlases for clinical applications. The atlases developed so far in this group (along with new patented or patent-pending solutions) are exploratory to illustrate anatomic [16], functional [10, 14, 42, 43], and pathologic [15] variability.

\subsection{Atlas design principles}

Ten principles of atlas design are the following

1. Electronic The atlas is electronic (digital) to make its presentation, manipulation, enhancement, extension, automatic processing, and distribution easy.

2. $3 D$ The atlas is $3 \mathrm{D}$ and of high spatial resolution to avoid spatial inconsistency and sparseness. It provides both surface and sectional anatomy.

3. In vivo The atlas is created from in vivo advanced, multispectral MR, and high-resolution $\mathrm{CT}$ imaging to avoid post-mortem artifacts and spatial distortions resulting from specimen processing, particularly, histological sectioning.

4. Holistic/single specimen We aim to build a maximally complete model of the whole brain along with the head and neck (an anatomically holistic approach), in contrast to just any part or component of it (a mosaic approach). Any new component or (sub)system added to the existing model must precisely fit to it, which is 
facilitated (but not obviously feasible due to geometric imaging distortions and artifacts) by the use of a single, reference, rescannable brain specimen.

5. Top-down design The complexity of the cerebral model has been continuously increasing by using a top-down approach. The whole brain has been continuously subdivided into smaller and smaller components, and extended with more and more (sub)systems, and the head and neck have been included. Consequently, several versions of the atlas are available now, and new extended, versions will be progressively upcoming.

6. Deterministic/labelable model The boundaries between the neighboring components shall be uniquely determined to avoid (or minimized) neither holes nor overlaps between them and to make any location (on the 3D model and the MR scan) uniquely labelable (being named) or characterized with various features. Hence, these brain, head, and neck components form 3D "blocks".

7. Scalable design/from blocks to brain, head, and neck Both the model and the atlas user interface are scalable. The complete model, or any of its parts, is easily composed (assembled) from or decomposed (disassembled) into blocks at multiple levels with a few clicks of the mouse. The user interface is extendable to handle new modules.

8. Stereotactic reference The model is placed in a stereotactic (bicommissural) coordinate system and forms a stereotactic reference with coordinate readout.

9. Affordableleasy to use The atlas runs on a standard computer (PC and MAC) as well as on mobile platforms (iPad and Android-based). It is easy to operate, and any system/configuration can be created just with a few clicks.

10. Beautiful brain, head, and neck The atlas is designed with an emphasis on esthetics and, particularly, model coloring to make the brain, head, and neck as well as the user interface beautiful.

\subsection{Atlas content}

At present (i.e., included into the recent version [36]), the atlas of the brain, head, and neck contains about 3,000 following components:

- brain divided into the left and right hemispheres, cerebrum, cerebellum, and brainstem;

- cerebellum divided into the left and right hemispheres;

- brainstem divided into the left and right parts, and parcellated into the midbrain, pons, and medulla;

- cerebral cortex completely parcellated into lobes, gyri, and gyri with sulci as well as 3D Brodmann's areas (not integrated yet with [36]);
- spinal cord divided into the left and right parts;

- white matter parcellated into cerebral, posterior fossa, and deep white matter;

- gray matter nuclei completely parcellated;

- ventricular system completely parcellated;

- intracranial vasculature (arteries, veins, and dural sinuses) parcellated into more than 1,300 vessels, the smallest of $0.08 \mathrm{~mm}$ in diameter;

- intracranial arteries grouped into the internal carotid, anterior cerebral, middle cerebral, posterior cerebral, vertebral and basilar arteries, and the circle of Willis, each group completely parcellated;

- intracranial veins grouped into superficial, deep, and posterior fossa veins, each group completely parcellated;

- dural sinuses completely parcellated;

- extracranial vasculature grouped into arteries and veins, each group completely parcellated;

- white matter tracts grouped into associations, commissures, projections, and posterior fossa tracts, each group completely parcellated;

- cranial nerves $(\mathrm{CN})$ grouped into CN I-CN XII, each group completely parcellated, along with the nuclei with more than 630 components;

- head muscles grouped into extra-ocular, facial, masticatory, and other muscles, each group completely parcellated;

- glands grouped into mouth and other glands, each group completely parcellated;

- skull completely parcellated into all 29 bones;

- skin divided into the left and right parts;

- functional (auditory and visual) systems parcellated;

- cervical spine parcellated into vertebrae;

- pharynx parcellated (not integrated yet with [36]);

- teeth completely parcellated into all 32 teeth (not integrated yet with [36]).

This content is grouped in our recent atlas [36] into 17 modules (tissue classes): central nervous system, deep structures, ventricles, white matter, white matter tracts, intracranial arterial system, intracranial venous system, head muscles, glands, extracranial arteries, extracranial veins, skull, skin, neck, visual system, and auditory system.

\subsection{Atlas functionality}

The atlas provides a rich functionality for model manipulation and exploration. These functions are grouped into seven main clusters for.

1. Structure selection Select and/or deselect all, (tissue) classes clusters, classes, groups, individual structures, and/or left, right or both sides (during 3D scene compositing and/or decompositing operations). These operations are supported by two-way mapping between 
the $3 \mathrm{D}$ view (where the $3 \mathrm{D}$ model is displayed) and the anatomical index.

2. $3 D$ model real-time manipulation The composed 3D scene can be displayed in 3D and manipulated in real time by performing its rotation (arbitrarily or around any reference axis), zooming, and panning; setting predefined views (anterior, posterior, superior, inferior, left and right); and starting/stopping animation (3D scene auto rotation).

3. Virtual dissections The 3D model is dissectible by means of 3D cutting of the cerebrum, cerebellum, brainstem, spinal cord, white matter, and skull. Dissections are 3D by means of seven cutting planes: two planes (to cut the model from its both ends) per axial, coronal and sagittal orientations and an arbitrary plane in the viewing direction. This operation allows exposing structures lying inside (to see through the brain) as well as enables a simultaneous display of surface and sectional anatomy in any orientation.

4. Scan manipulation The main structural (MP-RAGE) scan can be shown along with the 3D model, and its axial, coronal, and sagittal planes scrolled and manipulated while being displayed in 3D.

5. Atlas content querying Similarly to a two-way (3D view-anatomical index) mapping, querying is also done in a two-way. Either the 3D scene or the index can be queried. When querying a structure in the 3D scene about its properties, its name is highlighted is the index and a label is shown in the 3D view with the name (and the diameter for the vessels). The labels can be placed permanently and manipulated in 3D. In general, a vector of items can be provided, such as literature materials or references, like in [29, 37] atlases. When querying the index about structure location, the structure (or a group of structures) is (are) highlighted in the 3D view.

6. Coordinates and distance readout As the atlas is stereotactic, 3D coordinates of any location are provided and a distance between any two atlas points can be measured.

7. Supporting functions They provide image saving, clearing labels and highlights, and getting information and help.

\section{Results}

To date, we have developed 35 brain atlases for education [20], [44, 45], research [18, 46] and clinical applications [47], mainly in neuroradiology $[28,48,49]$ neurosurgery $[10,50$, 51], and neurology [39-41]. Dedicated atlas-based solutions have been proposed and developed for stereotactic and functional neurosurgery $[10,19,42,43,50,52-54]$, stroke image analysis and prediction $[15,55]$, scan interpretation [45], brain cancer [49], psychiatry (schizophrenia) [56], and human brain mapping [21, 46, 51]. We have extensively published these efforts in about 200 publications, including journal and conference papers as well as clinical abstracts.

Our atlases have been (1) licensed to 63 companies and institutions globally; (2) installed in over 1,500 surgical workstations by major surgical companies (including Medtronic/USA, Brainlab/Germany and Elekta/Sweden); (3) distributed to medical schools and individual clinicians by a leading medical publisher Thieme (over 7,500 copies of 15 atlases, some of them bestsellers); (4) made available publicly to numerous medical societies (e.g., World Stroke Organization, Society for Brain Mapping and Therapeutics), organizations, and individuals; (5) globally recognized receiving 23 awards, where 20 awards are from leading medical societies, including top ones (Summa cum laude in 1997, 2008, 2012, and 2014 from American Society of Neuroradiology and Magna cum Laude (considered a Radiological Oscar) with Excellence in Design in 2009 from Radiological Society of North America); and (6) featured on the Discovery Chan$n e l, C N N$, and in The Wall Street Journal. We have introduced electronic brain atlases to stereotactic and functional neurosurgery $[10,54]$, mainly for deep brain stimulation to treat Parkinson's disease and other disorders. Hundreds of thousands of patients have been treated by employing our atlases.

The holistic and reference atlas constructed to date contains the brain, head, and neck with about 3,000 components and is made available [36]. The virtual model in the atlas contains structure [17], intracranial vasculature [57], white matter tracts [58], cranial nerves with nuclei [59], head muscles and glands [60], extracranial vasculature [61], and a complete skull added recently reconstructed from a highresolution CT scan [62], each validated against the state-ofthe-art literature.

The images below illustrate the currently developed atlas: user interface (Fig. 2), cortical parcellation (Fig. 3), cerebrovasculature (Fig. 4), cranial nerves with white matter tracts (Fig. 5), skull, cervical spine, and extracranial vessels (Fig. 6), head muscles and glands (Fig. 7), and brain cutting and labeling (Fig. 8).

\section{Discussion}

Our atlas work is impactful despite being of a small funding scale. We have developed 35 commercial atlases and additionally numerous research prototypes presented at clinical and educational meetings. These brain atlases have a wide range of use. We have already developed atlas- 


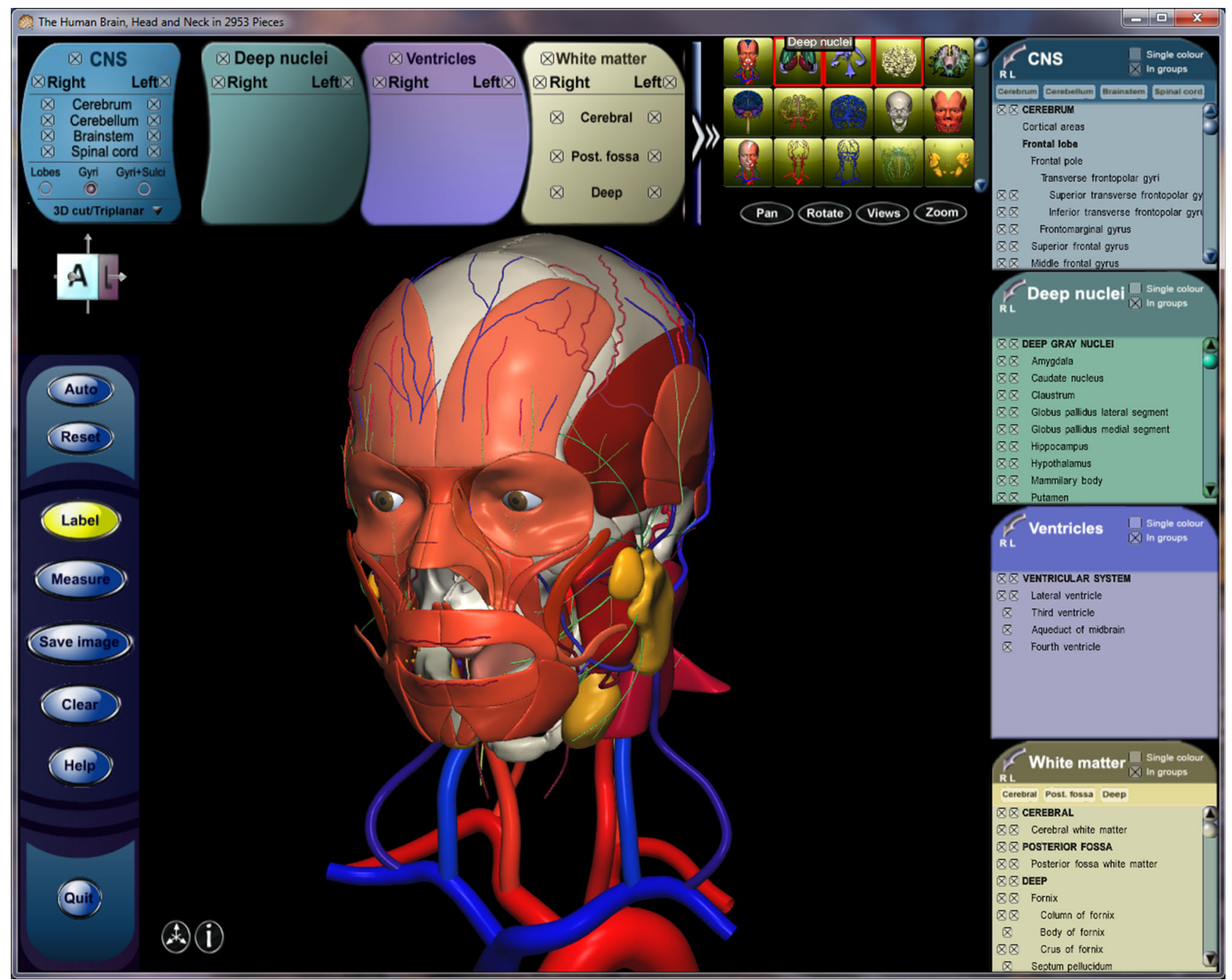

Fig. 2 User interface of the 2953 atlas: controls, anatomical index, and 3D view with the brain, head, and neck model

based applications ranging from education to research to clinical use.

\subsection{Advantages}

Our approach to brain atlas creation has several advantages. It leads to a construction of an anatomically holistic brain model, much more complete and detailed than any existing anatomical brain models created so far (to our best knowledge). Building it from in vivo data eliminates postmortem artifacts and spatial distortions resulting from histological sectioning. The use of a single, rescannable (over long time) specimen allows us to build a spatially consistent model, easily expandable with new scans, benefiting this way of fast developments in diagnostic imaging. A modular design provides an expandable content and scalable user interface. Virtual brain dissection enables the user to see, e.g., cortical areas hidden in the folded cortex, vessels cursing within the sulci, and deep structures with the surrounding parenchyma. 3D labeling enables the user to interactively explore the labeled cerebral model or any part of it with the labels attached to the components while being manipulated. The compositing and decompositing mechanism (from blocks to brain) allows the user to build any region of interest or (sub)system as well as to present local anatomy within its global context with a few clicks of mouse. The labelable triplanar display (especially useful for neuroradiology residents and neuroradiologists) merges sectional with surface anatomy. Mapping of the atlas to patient's data can be done using standard techniques for MR-to-MR registration. Then, the deformation field calculated for the structural scan of the brain specimen shall be applied to the corresponding 3D cerebral model. We have designed the atlases to run on a standard computer and mobile platforms (including iPad [34] and Androidbased [35]) in order to make them affordable to a wide 

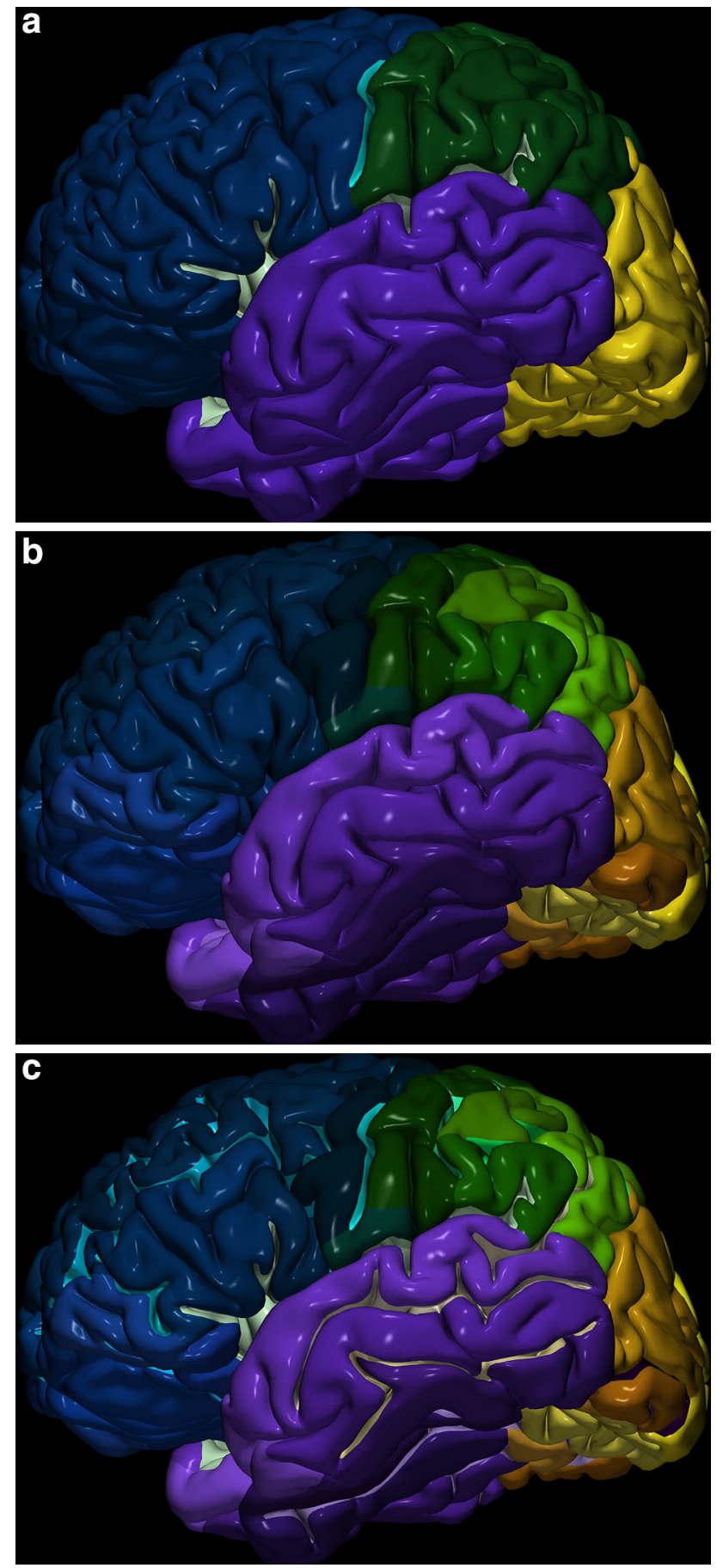

Fig. 3 Cortical parcellation: a lobes; b gyri; c gyri and sulci

spectrum of users ranging from a layman to researcher to medical student to clinician. By extending the model to the head and neck, the applicability of the atlas is expanded.

The atlases of neurologic disorders, combining neuroanatomy, neuroradiology, and neurology, are supportive in a recent trend in radiology (from volume- to value-based) aiming to enhance neuroradiologist-clinicians and neuroradiologist-patient communication. For instance, communicating
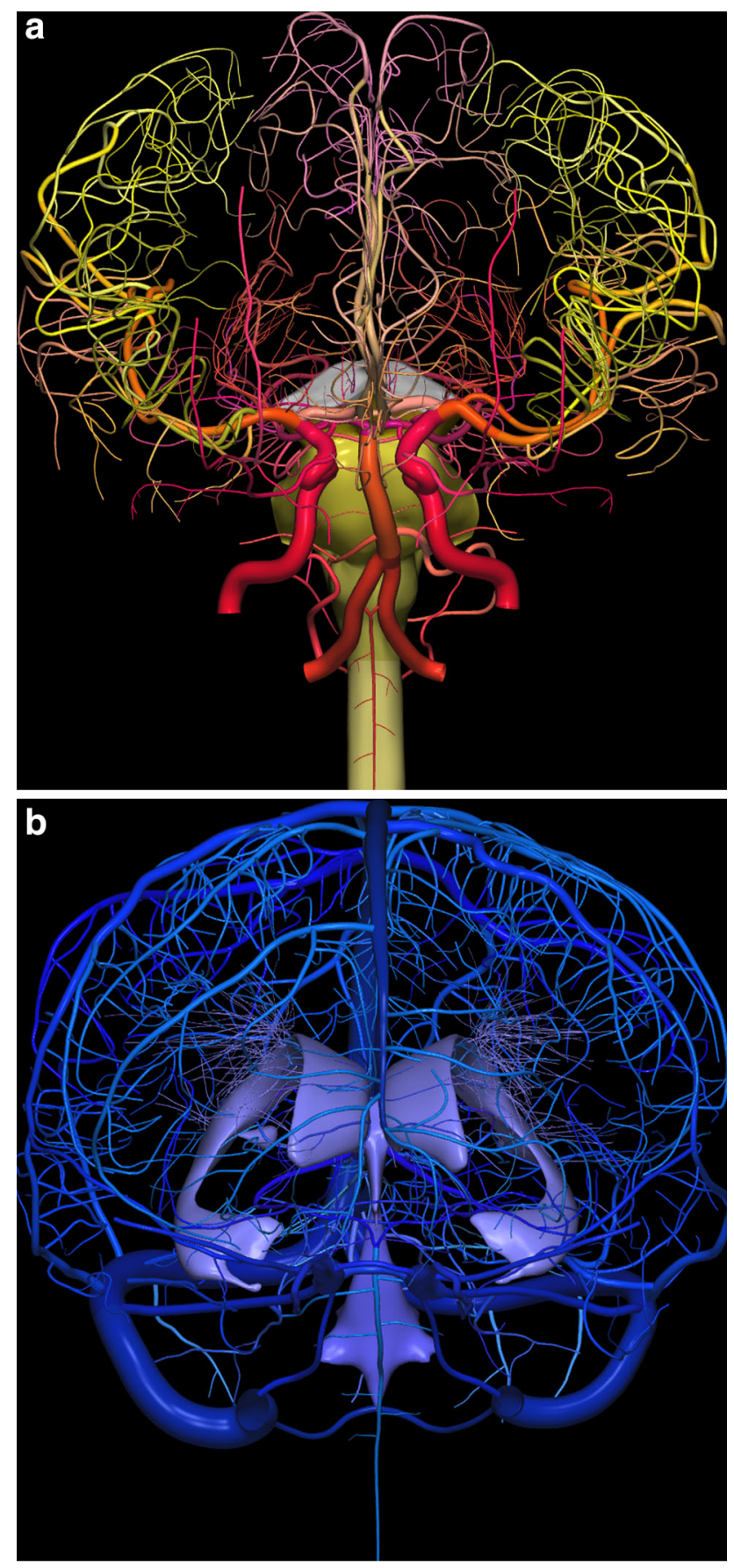

Fig. 4 Cerebrovasculature: a arterial system (along with the brainstem and spinal cord); b venous system (along with the ventricles)

a stroke situation is complicated and time consuming in a time critical condition and new tools are needed for this purpose. Then, the Stroke Atlas [41] may serve as an aid in patient-doctor communication facilitating to explain the situation to the patient and/or his or her family. Moreover, as this stroke atlas is self-explanatory and easy to use, it enables a layman to get familiarized with normal neuroanatomy and understand what happens in stroke. 


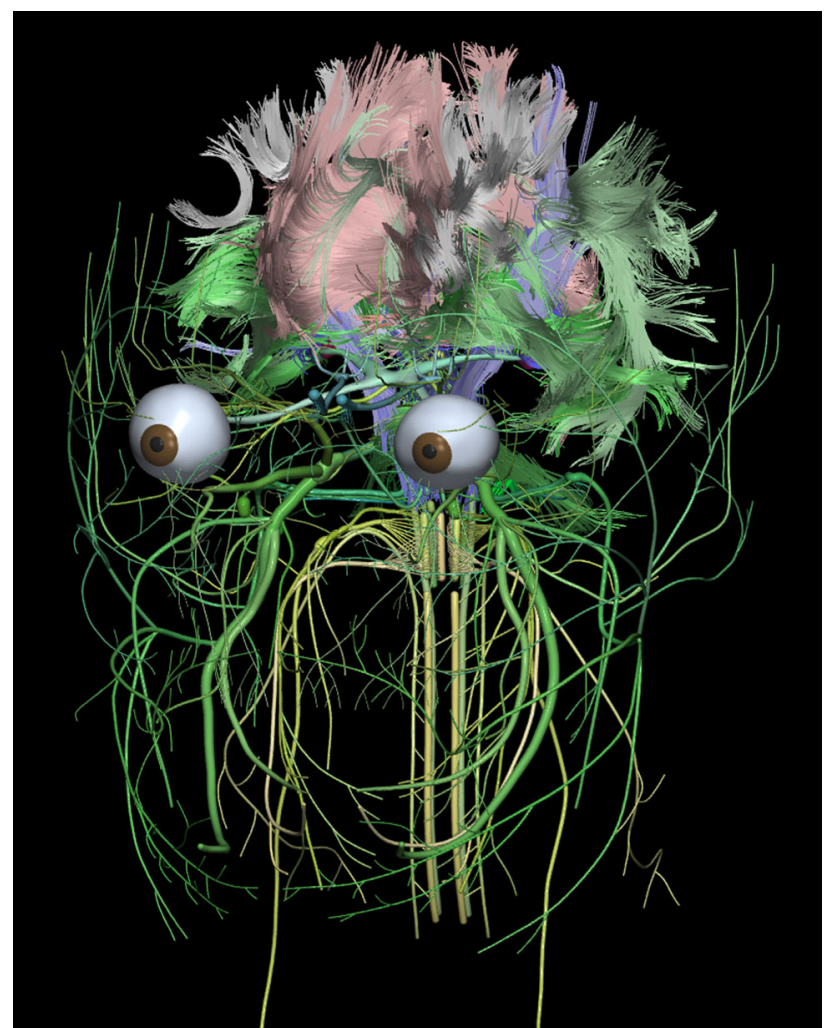

Fig. 5 Cranial nerves along with white matter tracts and visual system

\subsection{Deterministic versus population-based atlases}

Population-based atlases are particularly useful in anatomical interpretation of functional imaging observations (especially to highlight the uncertainty regions), studying intersubject variability and left-right asymmetries as well as they can distinguish abnormalities from normal variants, and detect group-specific features and average patterns. Moreover, dynamic atlases with time-varying data help analyze the growth rate and lifelong normal changes [13].

As both deterministic and population-based atlases have their own merits, their combination is advantageous as illustrated, for instance, by us in [52], where a highly anatomically parcellated atlas of the deep structures is integrated with a low parcellation, high spatial resolution, and population-based atlas of the subthalamic nucleus applicable for deep brain stimulation. Then, the population-based atlas facilitates to define the target (the point with the highest probability), while the anatomical atlas (containing target's anatomical neighborhood) the trajectory to it.

The averaging process, typically applied to construct population-based atlases, washes away the actual anatomical information. Consider, for instance, arterial variants of the circle of Willis (CoW). Averaging of the variants of an incomplete $\mathrm{CoW}$ in 3D results in a complete averaged

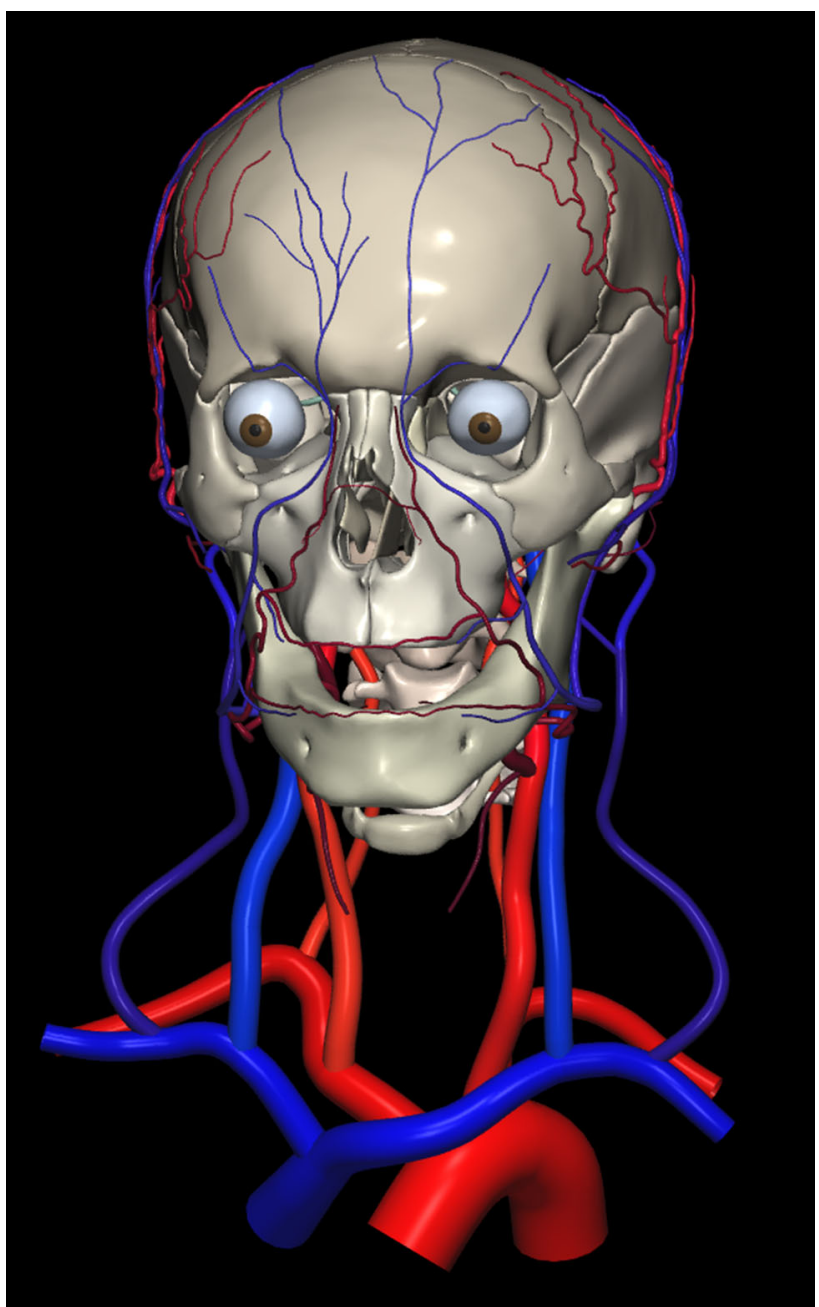

Fig. 6 Skull, cervical spine, extracranial arteries, extracranial veins, and visual system

CoW with lost information on variants' variability. To avoid this problem in a population-based atlas, we have proposed to include all the variants and present individually each of them in the context of a single reference deterministic atlas [16]. These examples illustrate that a detailed anatomical atlas is a critical component, independent of and not replaceable by a population-based atlas.

\subsection{Limitations}

Most of the current limitations are probably because this work is in progress. Our initial focus has been to develop an accurate and detailed anatomic model. This is because half of knowledge in several disciplines is neuroanatomy, including neurosurgery, neurology, neuroradiology, and neuroscience. Moreover, neuroanatomy and neuroscience are reported most difficult in medical education. With the future developments, as discussed below, the power and usefulness of the reference atlas will be growing. 


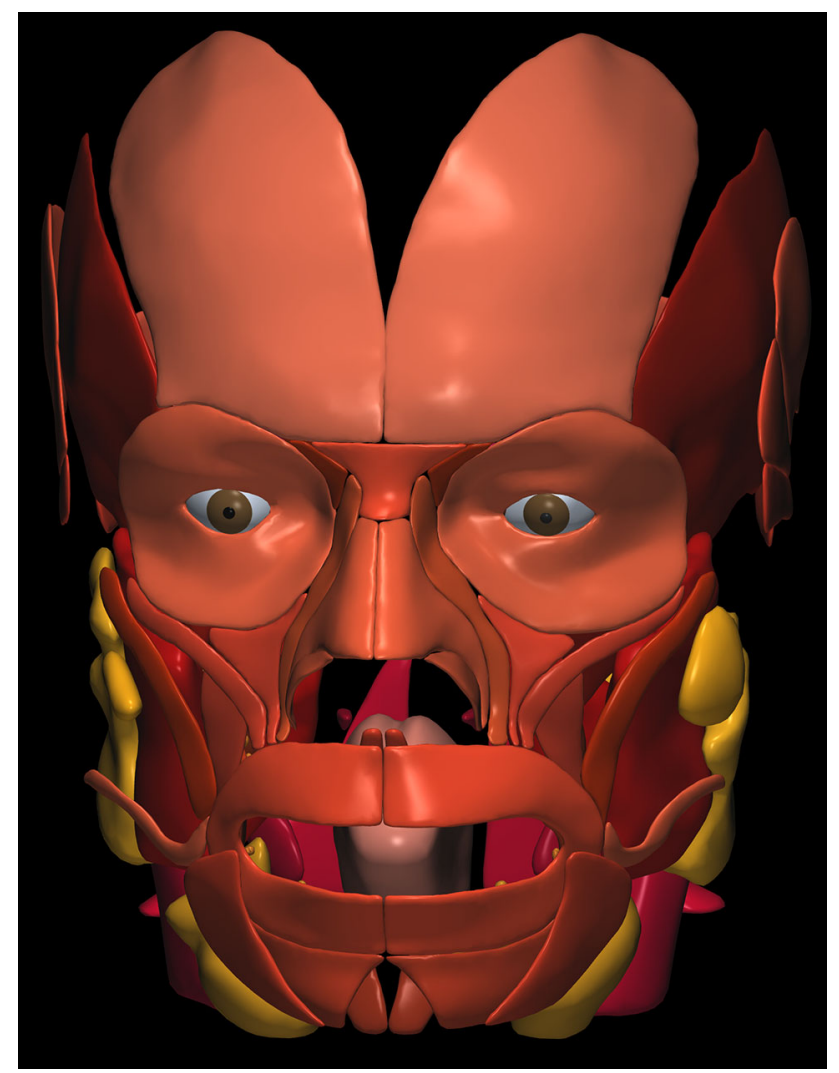

Fig. 7 Head muscles, glands, and visual system

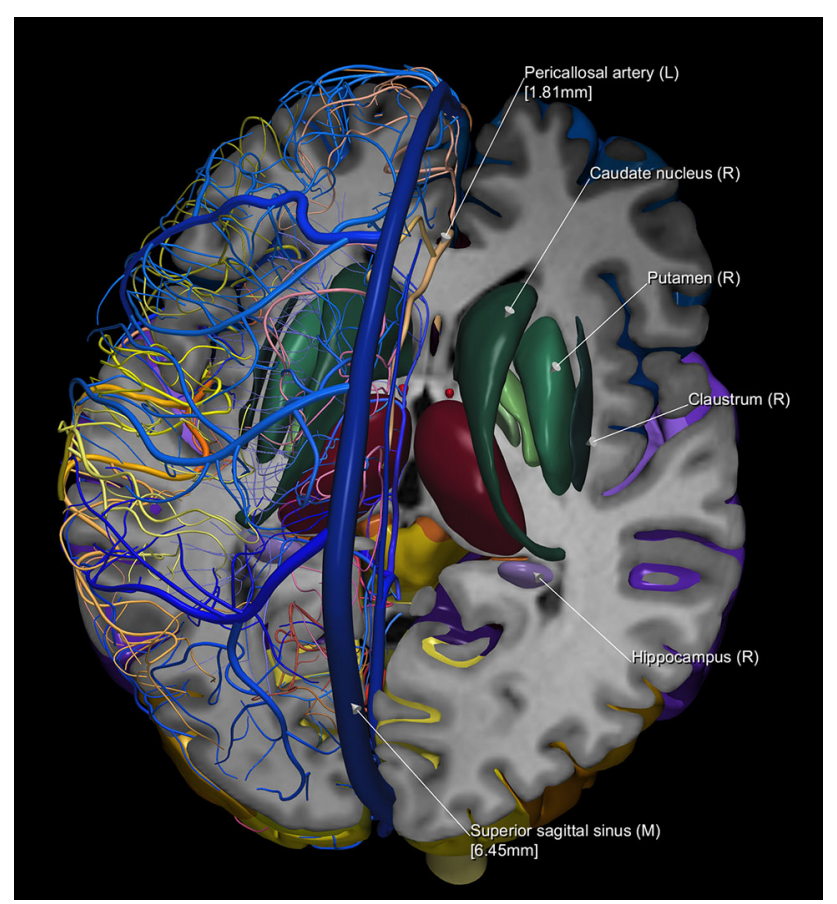

Fig. 8 Illustration of brain cutting and labeling

\subsection{Future developments}

As stated by Dr. Anne Osborn in the foreword to the 1492 atlas [31]: "you hold the future in your hands" and "applications of this tool are exciting to contemplate." Our design facilitates construction of a family of various atlases, such as application-specific, patient-oriented, layman-oriented, and medical speciality-oriented. This modular and scalable atlas can easily be extended by acquiring new scans of the same specimen, integrating new studies and simulations mapped to the same stereotactic atlas space, and by synthesizing small components which are beyond the current imaging resolution. Therefore, we keep brain scanning, segmenting, modeling, editing, cerebral model cutting, and structure synthesizing. We plan to integrate imaging (including structural, functional, connectional, and molecular images) and non-imaging data, literature materials, and simulation results, thereby growing the current atlas both "up" and "down." This effort may take decades to create a multi-level molecular, subcellular, cellular, circuital, anatomical, physiological, and behavioral brain atlas platform (provided that funding is available).

The 3D atlas of neurologic disorders [37] can easily be extended by adding many more synthesized lesions and linking them with clinical cases. This may expand the applicability of the atlas to become an aid for neurological diagnosis and pathology localization, particularly, in the radiology deprived regions, where diagnostic imaging is neither available nor affordable

\subsection{Usefulness in big brain projects}

This atlas is potentially useful in the recent big brain projects, including the BRAIN Initiative and The Human Brain Project. It can serve as a (1) reference for enormous amounts of data that will be generated; (2) framework for result integration and interpretation; (3) vehicle to present and disseminate the discoveries from science (and also within scientific disciplines) to medicine to public; (4) potential "Wikipedia" for the brain community; and (5) tool reducing a difficulty barrier (as the brain model is made easy and beautiful) in order to train a new wave of neurotechnologists and neuroscientists, make neurologic disorders more understandable, and educate public.

\subsection{Summary}

We have developed to date 35 commercial brain atlases (along with numerous research prototypes), licensed to 63 companies and institutions, and made available to medical 
societies, organizations, medical schools, and individuals. These atlases have been applied in education, research, and clinical applications, mainly in neuroradiology, neurosurgery, and neurology. Dedicated atlas-based solutions have been proposed and developed for stereotactic and functional neurosurgery, stroke image analysis, scan interpretation, brain cancer, psychiatry (schizophrenia), and human brain mapping. Hundreds of thousands of patients have been treated by using our atlases.

Our ultimate objective is to create a holistic, reference, and extendable atlas of the whole adult human brain along with the head and neck. The first version of such an atlas has been developed and made available. The atlas has been created from multispectral 3 and $7 \mathrm{~T}$ and high-resolution $\mathrm{CT}$ in vivo scans. It is fully $3 \mathrm{D}$, scalable, interactive, and highly detailed with about 3,000 labeled components.

This atlas forms a foundation for the development of a multi-level molecular, cellular, anatomical, physiological, and behavioral brain atlas platform.

Acknowledgments The atlas development work was funded by ASTAR, Singapore. The author is very grateful to numerous individuals (listed as the co-authors in the references) who contributed to the atlas development.

Open Access This article is distributed under the terms of the Creative Commons Attribution License which permits any use, distribution, and reproduction in any medium, provided the original author(s) and the source are credited.

\section{References}

1. Underwood E (2013) Neuroscience. Brain project draws presidential interest, but mixed reactions. Science 339(6123):1022-1023. doi:10. 1126/science.339.6123.1022

2. Miller G (2011) Blue brain founder responds to critics, clarifies his goals. Sci Mag 34:748-749

3. Abbott A (2003) A new atlas of the brain. Nature 424:249-250

4. Hawrylycz MJ, Lein ES, Guillozet-Bongaarts AL, Shen EH, Ng L, Miller JA, van de Lagemaat LN, Smith KA, Ebbert A, Riley ZL, Abajian C, Beckmann CF, Bernard A, Bertagnolli D, Boe AF, Cartagena PM, Chakravarty MM, Chapin M, Chong J, Dalley RA, Daly BD, Dang C, Datta S, Dee N, Dolbeare TA, Faber V, Feng D, Fowler DR, Goldy J, Gregor BW, Haradon Z, Haynor DR, Hohmann JG, Horvath S, Howard RE, Jeromin A, Jochim JM, Kinnunen M, Lau C, Lazarz ET, Lee C, Lemon TA, Li L, Li Y, Morris JA, Overly CC, Parker PD, Parry SE, Reding M, Royall JJ, Schulkin J, Sequeira PA, Slaughterbeck CR, Smith SC, Sodt AJ, Sunkin SM, Swanson BE, Vawter MP, Williams D, Wohnoutka P, Zielke HR, Geschwind DH, Hof PR, Smith SM, Koch C, Grant SG, Jones AR (2012) An anatomically comprehensive atlas of the adult human brain transcriptome. Nature 489(7416):391-399

5. Sunkin SM, Ng L, Lau C, Dolbeare T, Gilbert TL, Thompson CL, Hawrylycz M, Dang C (2013) Allen Brain Atlas: an integrated spatio-temporal portal for exploring the central nervous system. Nucleic Acids Res 41(D1):D996-D1008

6. A.D.A.M (1996) Animated dissection of anatomy for medicine. User's Guide, A.D.A.M, Atlanta
7. Nowinski WL, Thirunavuukarasuu A (2004) The cerefy clinical brain atlas on CD-ROM. Thieme, New York

8. Sundsten JW, Brinkley JF, Eno K, Prothero J (1994) The digital anatomist. Interactive Brain Atlas. CD ROM for the Macintosh. University of Washington, Seattle

9. Hoehne KH (2001) Voxel-man, brain and skull. version 2.0. Springer, Heidelberg

10. Nowinski WL (2009) Anatomical and probabilistic functional atlases in stereotactic and functional neurosurgery. In: Lozano A, Gildenberg P, Tasker R (eds) Textbook of stereotactic and functional neurosurgery. Springer, New York, pp 395-441

11. Amunts K, Schleicher A, Zilles K (2007) Cytoarchitecture of the cerebral cortex-more than localization. Neuroimage 37(4): 1061-1065

12. Mazziotta J, Toga A, Evans A, Fox P, Lancaster J, Zilles K, Woods R, Paus T, Simpson G, Pike B, Holmes C, Collins L, Thompson P, MacDonald D, Iacoboni M, Schormann T, Amunts K, Palomero-Gallagher N, Geyer S, Parsons L, Narr K, Kabani N, Le Goualher G, Boomsma D, Cannon T, Kawashima R, Mazoyer B (2001) A probabilistic atlas and reference system for the human brain: international consortium for brain mapping (ICBM). Philos Trans R Soc Lond B 356(1412):1293-1322

13. Toga AW, Thompson PM, Mori S, Amunts K, Zilles K (2006) Towards multimodal atlases of the human brain. Nat Rev Neurosci 7(12):952-966

14. Nowinski WL, Belov D, Benabid AL (2003) An algorithm for rapid calculation of a probabilistic functional atlas of subcortical structures from electrophysiological data collected during functional neurosurgery procedures. Neuroimage 18(1):143-155

15. Nowinski WL, Gupta V, Qian GY, Ambrosius W, Kazmierski R (2014) Population-based stroke atlas for outcome prediction: method and preliminary results for ischemic stroke from CT. PLoS One. August 14; 9(8):e102048. doi: 10.1371/journal.pone. 0102048. eCollection 2014; http://www.plosone.org/article/ info\%3Adoi\%2F10.1371\%2Fjournal.pone.0102048

16. Nowinski WL, Thirunnavuukarasuu A, Volkau I, Marchenko Y, Aminah B, Puspitasaari F, Runge VM (2009) A three-dimensional interactive atlas of cerebral arterial variants. Neuroinformatics 7(4):255-264

17. Nowinski WL, Chua BC, Qian GY, Nowinska NG (2012) The human brain in 1700 pieces: design and development of a threedimensional, interactive and reference atlas. J Neurosci Methods 204(1):44-60

18. Nowinski WL (1999) Analysis of medical images by means of brain atlases. Mach Graph Vis 8(3):449-468

19. Nowinski WL, Thirunavuukarasuu A, Benabid AL (2005) The cerefy clinical brain atlas. Extended edition with surgery planning and intraoperative support. Thieme, New York

20. Nowinski WL, Thirunavuukarasuu A, Bryan RN (2002) The cerefy atlas of brain anatomy. An introduction to reading radiological scans for students, teachers, and researchers. Thieme, New York

21. Nowinski WL, Thirunavuukarasuu A, Kennedy DN (2000) Brain atlas for functional imaging. Clinical and research applications. Thieme, New York

22. Nowinski WL, Bryan RN, Raghavan R (1997) The electronic clinical brain atlas. Multiplanar navigation of the human brain. Thieme, Stuttgart

23. Talairach J, Tournoux P (1988) Co-planar stereotactic atlas of the human brain. Georg Thieme Verlag/Thieme Medical Publishers, Stuttgart

24. Schaltenbrand G, Wahren W (1977) Atlas of stereotaxy of the human brain. Georg Thieme Verlag, Stuttgart

25. Talairach J, Tournoux P (1993) Referentially oriented cerebral MRI Anatomy. Atlas of stereotaxic anatomical correlations for 
gray and white matter. Georg Thieme Verlag/Thieme Medical Publishers, Stuttgart

26. Ono M, Kubik S, Abernathey CD (1990) Atlas of the cerebral sulci. Georg Thieme Verlag/Thieme Medical Publishers, Stuttgart

27. Nowinski WL, Fang A, Nguyen BT, Raphel JK, Jagannathan L, Raghavan R, Bryan RN, Miller G (1997) Multiple brain atlas database and atlas-based neuroimaging system. Comput Aided Surg 2(1):42-66

28. Nowinski WL: Electronic brain atlases: features and applications. In: 3D image processing: techniques and clinical applications (eds. Caramella D, Bartolozzi C). Medical Radiology series, Springer, Berlin 2002:79-93

29. Nowinski WL, Thirunavuukarasuu A, Volkau I, Marchenko Y, Runge VM (2009) The cerefy atlas of cerebral vasculature. Thieme, New York

30. Nowinski WL, Chua BC, Qian GY, Marchenko Y, Puspitasari F, Nowinska NG, Knopp MV (2011) The human brain in 1492 pieces: structure, vasculature, and tracts (version 1.1 for MAC). Thieme, New York

31. Nowinski WL, Chua BC, Qian GY, Marchenko Y, Puspitasari F, Nowinska NG, Knopp MV (2011) The human brain in 1492 pieces: structure, vasculature, and tracts. (version 1.0 for $\mathrm{PC}$ ). Thieme, New York

32. Nowinski WL, Chua BC (2014) The human brain in 1969 pieces: structure, vasculature, tracts, cranial nerves, systems, head muscles, and glands (version 2.0). Thieme, New York

33. Nowinski WL, Chua BC, Qian GY, Nowinska NG (2013) The human brain in 1969 pieces: structure, vasculature, tracts, cranial nerves, and systems. Thieme, New York

34. Nowinski WL, Chua BC The complete human brain (version 1.0 for iPad). Thieme, New York, 2013/AppStore

35. Nowinski WL, Chua BC, Ngai V (2014) The 3D brain atlas (version 1.0 for Android tablet). Thieme, New York

36. Nowinski WL, Chua BC, Thaung TSL, Wut Yi SH (2015) The human brain, head and neck in 2953 pieces. Thieme, New York

37. Nowinski WL, Chua BC, Wut Yi SH (2014) 3D atlas of neurologic disorders (version 1.0 for PC/MAC). Thieme, New York

38. Nowinski WL, Chua BC, Ngai V 3D atlas of neurologic disorders (version 1.0 for iPad). Thieme, New York, 2013/AppStore

39. Nowinski WL, Chua BC (2013) Three-dimensional interactive atlas of cranial nerve-related disorders. Neuroradiol $\mathrm{J}$ 26(3):263-275

40. Nowinski WL, Chua BC (2013) Bridging neuroanatomy, neuroradiology and neurology: three-dimensional interactive atlas of neurological disorders. Neuroradiol J 26(3):252-262

41. Nowinski WL, Chua BC (2013) Stroke Atlas: a 3D interactive tool correlating cerebrovascular pathology with underlying neuroanatomy and resulting neurological deficits. Neuroradiol $\mathbf{J}$ 26(1):56-65

42. Nowinski WL, Belov D, Thirunavuukarasuu A, Benabid AL (2006) A probabilistic functional atlas of the VIM nucleus constructed from pre-, intra- and post-operative electrophysiological and neuroimaging data acquired during the surgical treatment of Parkinson's disease patients. Stereotact Funct Neurosurg 83(5-6):190-196

43. Nowinski WL, Belov D, Pollack P, Benabid AL (2005) Statistical analysis of 168 bilateral subthalamic nucleus implantations by means of the probabilistic functional atlas. Neurosurgery 57(4 Suppl):319-330

44. Nowinski WL, Thirunavuukarasuu A, Ananthasubramaniam A, Chua BC, Qian G, Nowinska NG, Marchenko Y, Volkau I (2009) Automatic testing and assessment of neuroanatomy using a digital brain atlas: method and development of computer- and mobile-based applications. Anat Sci Educ 2(5):244-252

45. Nowinski WL, Thirunavuukarasuu A, Volkau I, Marchenko Y, Aminah B, Gelas A, Huang S, Lee LC, Liu J, Ng TT, Nowinska
N, Puspitasari F, Qian G, Runge VM (2009) A new presentation and exploration of human cerebral vasculature correlated with surface and sectional neuroanatomy. Anat Sci Educ 2(1):24-33

46. Nowinski WL, Thirunavuukarasuu A (2001) Atlas-assisted localization analysis of functional images. Med Image Anal 5(3):207-220

47. Nowinski WL (2007) Human brain atlases in education, research and clinical applications. In: Wu JL, Ito K, Tobimatsu S, Nishida $\mathrm{T}$, Fukuyama H (eds) Complex medical engineering. Springer, Tokyo, pp 335-349

48. Nowinski WL, Qian G, Bhanu Prakash KN, Hu Q, Aziz A (2006) Fast Talairach Transformation for magnetic resonance neuroimages. J Comput Assist Tomogr 30(4):629-641

49. Nowinski WL, Belov D (2005) Towards atlas-assisted automatic interpretation of MRI morphological brain scans in the presence of tumor. Acad Radiol 12:1049-1057

50. Nowinski WL, Chua BC, Volkau I, Puspitasari F, Marchenko Y, Runge VM, Knopp MV (2010) Simulation and assessment of cerebrovascular damage in deep brain stimulation using a stereotactic atlas of vasculature and structure derived from multiple 3T and 7T scans. J Neurosurg 113(6):1234-1241

51. Nowinski WL, Thirunavuukarasuu A (2003) A locus-driven mechanism for rapid and automated atlas-assisted analysis of functional images by using the brain atlas for functional imaging. Neurosurg Focus 15(1):Article 3

52. Nowinski WL, Thirunavuukarasuu A, Liu J, Benabid AL (2007) Correlation between the anatomical and functional human subthalamic nucleus. Stereotact Funct Neurosurg 85:88-93

53. Nowinski WL, Benabid AL (2002) New directions in atlas-assisted stereotactic functional neurosurgery. In: Germano IM (ed) Advanced techniques in image-guided brain and spine surgery. Thieme, New York, pp 162-174

54. Nowinski WL (2001) Computerized brain atlases for surgery of movement disorders. Semin Neurosurg 12(2):183-194

55. Nowinski WL, Qian G, Bhanu Prakash KN, Thirunavuukarasuu A, Hu QM, Ivanov N, Parimal AS, Runge VL, Beauchamp NJ (2006) Analysis of ischemic stroke MR images by means of brain atlases of anatomy and blood supply territories. Acad Radiol 13(8):1025-1034

56. Sim K, Yang GL, Loh D, Poon LY, Sitoh YY, Verma S, Keefe R, Collinson S, Chong SA, Heckers S, Nowinski WL, Pantelis C (2009) White matter abnormalities and neurocognitive deficits associated with the passivity phenomenon in schizophrenia: a diffusion tensor imaging study. Psychiatry Res 172(2):121-127. doi:10.1016/j.pscychresns.2009.02.003

57. Nowinski WL, Chua BC, Puspitasari F, Volkau I, Marchenko Y, Knopp MV (2011) Three-dimensional reference and stereotactic atlas of human cerebrovasculature from 7 Tesla. Neuroimage 55(3):986-998

58. Nowinski WL, Chua BC, Yang GL, Qian GY (2012) Three-dimensional interactive human brain atlas of white matter tracts. Neuroinformatics 10(1):33-55

59. Nowinski WL, Johnson A, Chua BC, Nowinska NG (2012) Three-dimensional interactive and stereotactic atlas of cranial nerves and nuclei correlated with surface neuroanatomy, vasculature and magnetic resonance imaging. J Neurosci Methods 206(2):205-216

60. Nowinski WL, Chua BC, Johnson A, Qian G, Poh LE, Yi SHW, Aminah B, Nowinska NG (2013) Three-dimensional interactive and stereotactic atlas of head muscles and glands correlated with cranial nerves and surface and sectional neuroanatomy. J Neurosci Methods 215(1):12-18

61. Nowinski WL, Thaung TSL, Chua BC, Wut Yi SH, Yang Y, Urbanik A (2015) Three-dimensional stereotactic atlas of the extracranial vasculature correlated with the intracranial vasculature, cranial nerves, skull and muscles. Neuroradiol J 
62. Nowinski WL, Thaung TSL, Chua BC, Wut Yi SH, Ngai V, Yang Y, Chrzan R, Urbanik A (2015) Three-dimensional stereotactic atlas of the adult human skull correlated with the brain, cranial nerves and intracranial vasculature. J Neurosci Methods (in press)

Wieslaw L. Nowinski is an affiliate professor at the Department of Radiology, University of Washington, Seattle, WA. His research includes brain atlases, stroke, deep brain stimulation, brain quantification, neuroinformatics, medical image processing, virtual reality, computer-assisted diagnosis and treatment, and future directions in computer-aided radiology and surgery. He has 543 publications, filed 51 patent applications ( 32 already granted, 15 in US and 8 Europe (EP)), and developed with his team 35 brain atlas products that are used worldwide in neurosurgery, neuroradiology, neurology, brain mapping, and neuroeducation. These atlases have been licensed to 63 companies and institutions. Fifteen brain atlases have been distributed by Thieme Medical Publishers, New York-Stuttgart. The atlases are also installed in 1,500 surgical workstations. He has been conferred with 42 awards and honors; 25 awards are from leading medical societies, including Pioneer in Medicine in 2013 from Society for Brain Mapping \& Therapeutics; Magna cum Laude (radiological Oscar) from Radiological Society of North America in 2009 and 2004; Magna cum Laude from European Congress of Radiology in 2000; Summa cum Laude in 2014, 2012, 2008, and 1997; and Magna cum Laude in 2009 and 2005 from American Society of Neuroradiology. He has been an Asian Innovation Awards 2010 finalist (for his stroke work) and in top 3 nominees for European Inventor Award 2014 organized by the European Patent Office in category of lifetime achievement (for his brain atlas work). He has been named The Outstanding Pole (in the world) in 2012. His work has been featured in The Wall Street Journal and on The Discovery Channel, CNN, and Channel News Asia, among others. 